\title{
Cascading optofluidic phase modulators for performance enhancement in refractive adaptive optics
}

\author{
Pouya Rajaeipour $\odot,{ }^{*}$ Kaustubh Banerjee $\odot$, Alex Dorn, Hans Zappe $\odot$, and Çağlar Ataman $\odot$ \\ University of Freiburg, Gisela and Erwin Sick Laboratory for Micro-Optics, Department of Microsystems Engineering, Freiburg, Germany
}

\begin{abstract}
We discuss the implementation and performance of an adaptive optics (AO) system that uses two cascaded deformable phase plates (DPPs), which are transparent optofluidic phase modulators, mimicking the common woofer/tweeter-type astronomical AO systems. One of the DPPs has 25 electrodes forming a keystone pattern best suited for the correction of low-order and radially symmetric modes; the second device has 37 hexagonally packed electrodes better suited for high-order correction. We also present simulation results and experimental validation for a new open-loop control strategy enabling simultaneous control of both DPPs, which ensures optimum correction for both large-amplitude low-order, and complex combinations of low- and high-order aberrations. The resulting system can reproduce Zernike modes up to the sixth radial order with stroke and fidelity up to twice better than what is attainable with either of the DPPs individually. The performance of the new AO configuration is also verified in a custom-developed fluorescence microscope with sensorless aberration correction.
\end{abstract}

Keywords: adaptive optics; woofer/tweeter; phase modulation; optofluidics; aberration correction; open-loop control.

Received Jul. 11, 2020; revised manuscript received Oct. 6, 2020; accepted for publication Oct. 19, 2020; published online Nov. 18, 2020.

(C) The Authors. Published by SPIE and CLP under a Creative Commons Attribution 4.0 Unported License. Distribution or reproduction of this work in whole or in part requires full attribution of the original publication, including its DOI.

[DOI: 10.1117/1.AP.2.6.066005]

\section{Introduction}

Adaptive optics (AO) is an image correction technique that features a dynamically reconfigurable optical element to compensate for sample-, system-, or medium-induced wavefront aberrations. ${ }^{1,2}$ Commonly using a deformable mirror (DM) or a liquid crystal spatial light modulator (LC-SLM), it is predominantly the capabilities of this component that limit the quality of aberration correction, and thus the overall optical system performance. Modulators developed for large stroke are usually limited in spatial frequency of the correction, while the ones intended for high orders can be limited by the available stroke (as the only available exceptions can be referred to the high-end electromagnetic-actuated $\mathrm{DMs}^{3}$ ).

Using two separate modulators for low- and high-order correction, a configuration referred to as a woofer/tweeter (W/T) $\mathrm{AO}^{4-7}$ can be effective in overcoming this trade-off. Several

*Address all correspondence to Pouya Rajaeipour, pouya.rajaeipour@imtek.unifreiburg.de examples of successful W/T implementations can be found in the literature for $\mathrm{AO}$ in ground-based telescopes, ${ }^{4,8,9}$ ophthalmology applications, ${ }^{6,10}$ and laser micro-fabrication. ${ }^{11,12}$ In AO microscopy, a particularly active recent research field, notable examples were demonstrated by Wright et al. ${ }^{13}$ and Li et al. ${ }^{7}$ The former is a closed-loop system for dynamic focus tracking using a spatial light modulator and a DM within a confocal microscope, and the latter is a wide-field structured illumination microscope with a dual-DM AO system. ${ }^{7}$ The main factors inhibiting more widespread adoption of W/T AO are the hardware complexity due to the two reflective phase modulators at conjugate planes and their respective control.

We present here a new and particularly compact type of W/T AO employing multiple phase modulators in the form of two cascaded deformable phase plates (DPPs). The DPP is an optofluidic transmissive spatial phase modulator with a 2D array of electrostatic actuators. ${ }^{14}$ Compared to other transmissive phase modulators such as LC-SLMs, ${ }^{15}$ the DPP is higher in transmission efficiency, free from diffraction effects, and polarization independent. In contrast with multi-actuator lenses, ${ }^{16,17}$ which 
have a limited number of radial actuators circumscribing the optical aperture, the DPP has a 2D array of actuators across the aperture, leading to more actuators and a higher-order correction. Furthermore, in contrast to piezoelectrically actuated phase modulators, the DPP is free from hysteresis effects ${ }^{14,18}$ and does not require additional modeling or hardware for compensating these unwanted effects. ${ }^{19,20}$ These versatile characteristics make DPP applicable not only to W/T type multi-modulator AO systems, but also to the cascading of multiple identical modulators for simple stroke enhancement. An open-loop control system that treats the cascaded DPPs as a single phase modulator, while guaranteeing the optimum performance of each device regardless of their electrode count and distribution, is used. Using two closely positioned DPPs of contrasting electrode count and distribution, we experimentally demonstrate the advantage of this configuration in terms of amplitude, order, and fidelity of correcting up to the sixth radial order of Zernike modes. Finally, this $\mathrm{AO}$ system is also integrated into a custom fluorescence microscope to perform sensorless aberration correction for imaging micro-fluorescent beads.

\section{Cascaded Deformable Phase Plates}

Figure 1(a) depicts the simplified schematic of an AO implementation within a microscope that features two reflective phase modulators (such as DMs). ${ }^{7}$ In this configuration, each DM is individually conjugated to the pupil plane of the objective (or the pupil plane of the main optical system in the general case). Depending on the method used for controlling the DMs, an additional wavefront sensor can also be necessary to ensure optimal DM performance. Since each modulator folds the optical path and requires a dedicated set of relay lenses, the resulting complex system architecture is prone to increased losses and alignment errors. The optimum driving and control of the two DMs also pose a major challenge, as discussed in Sec. 3.

The new approach we consider here follows the configuration depicted in Fig. 1(b), where reflective DMs are replaced by refractive DPPs to eliminate the optical folds and reduce the relay lenses. Open-loop control of the DPPs facilitates the usage of a sensorless wavefront estimation algorithm. The resulting system is not only significantly more compact and robust, but it is also straightforward to integrate it into existing optical systems.

Figure 2(a) depicts the DPP, which is the core element enabling the practical serial cascading of phase modulators. It comprises an optofluidic chamber formed by a glass substrate supporting an array of transparent 2D electrodes, an annular spacer film, and a deformable membrane (made of polyimide) with a transparent conductive layer acting as the ground (a)

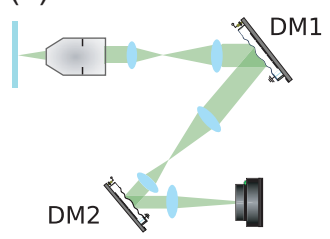

(b)

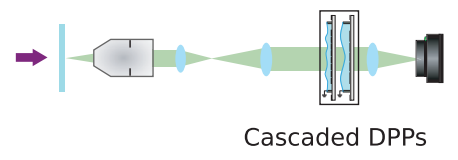

Fig. 1 (a) The conventional usage of two reflective elements for $A O$ versus (b) the cascaded configuration of two refractive DPPs. DM: deformable mirror. (a)
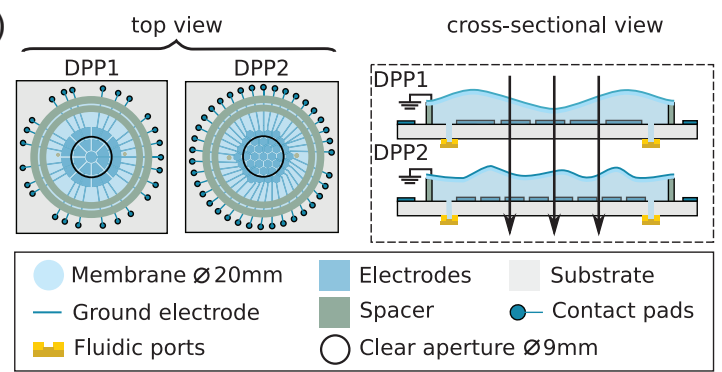

(b)

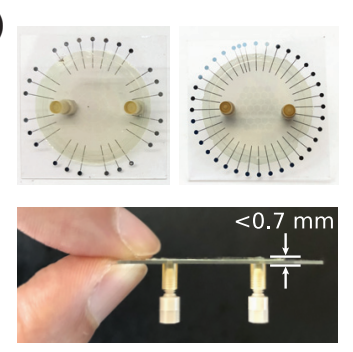

(c)

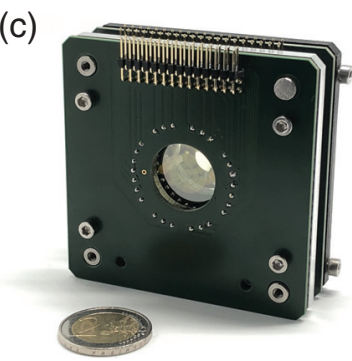

Fig. 2 (a) Schematics of the employed DPPs and their proposed cascaded configuration. DPP1 has 25 keystone-patterned electrodes that are distributed within an area of $12 \mathrm{~mm}$ in diameter, and DPP2 has 37 hexagonally patterned electrodes that can be circumscribed inside a circle of $12 \mathrm{~mm}$ in diameter. (b) Top and side views of the fabricated DPPs. (c) Stacked DPPs in their 60$\mathrm{mm}$ cage system compatible packaging and electrical interfacing.

electrode. The clear aperture of both DPPs, defined as the portion of the membrane used for phase modulation, is $9 \mathrm{~mm}$ and the fluidic chamber height is $110 \mu \mathrm{m}$. The fluidic interfaces are used to fill the chamber with an incompressible optical liquid, and subsequently sealed using PEEK microfluidic ports to avoid leakage. Applying a differential voltage between the embedded electrodes and the grounded membrane creates an attractive electrostatic force, which locally deforms the membrane leading to a spatial modulation of the optical path length across the clear aperture. The response time of the DPP is measured to be $<80 \mathrm{~ms},{ }^{14}$ limited by the compliance of the polymer membrane. Compared to state-of-the-art DMs, this speed can be a limiting factor for high-bandwidth applications, such as astronomical AO systems. Between 450 and 1100, the DDP has $>85 \%$ transmission, limited mostly by Fresnel losses from uncoated surfaces. The lower cutoff wavelength, on the other hand, is set by the strong absorption in the polyimide film below this range. With a thickness of $0.61 \mathrm{~mm}$ at the optical aperture, the DPP has limited dispersion, which would still be relevant for highly broadband applications. Due to its submillimeter thickness, the DPP is particularly suitable for multi-modulator wavefront shaping techniques. More details on the structure, operation principle, and experimental performance of the DPP can be found elsewhere. ${ }^{14,21}$

In this work, we used two variants of the DPP, one with 25 keystone-patterned electrodes (DPP1) and a second one with 37 hexagonally-patterned electrodes (DPP2). Figure 2(b) depicts the top and side views of both fabricated DPPs. With fewer electrodes and radial pseudo-symmetry of the electrode distribution, the DPP1 is optimized for lower-order (up to the fourth radial order) aberrations, while the DPP2 and its hexagonal densely packed electrodes can effectively replicate Zernike modes up to the sixth radial order. Figure 2(c) shows the 
cascaded DPPs in their packaging and electrical interfacing which is compatible with $60-\mathrm{mm}$ Thorlabs cage system.

\section{Open-Loop Control of Cascaded DPPs}

A major challenge for $\mathrm{AO}$ systems having multiple phase modulators at optically conjugated planes is their effective control such that they perform optimally for different aberration characteristics. The conventional approaches to this problem can be classified as sequential or simultaneous, depending on the addressing order of the two modulators. The former approach first addresses the woofer, which predominantly corrects for the low-order aberrations. Then the residual wavefront error, mostly comprising high spatial frequencies, is measured using a wavefront sensor, and subsequently corrected by the tweeter. ${ }^{5,22}$ This method has the potential for optimally driving each modulator, but requires a wavefront sensor. The simultaneous approach comes in two forms. By explicitly dividing the correction space between the two modulators by assigning different modes of a specific orthonormal set to each modulator, the operation of the two modulators can be decoupled. ${ }^{4,12,23,24}$ Alternatively, it is also possible to construct a single control matrix for both devices using their individual control matrices. ${ }^{25,26}$ Both methods can be operated in open loop without a wavefront sensor, but can result in suboptimal performance of the modulators due to lack of having a common space of correction modes in the former approach, and possible cross-correlations between the influence functions of each modulator electrode in the latter.

The approach developed here differs from all the above in that it includes:

- simultaneous and real-time control of multiple modulators, either with identical or distinctive features;

- open-loop calculation of the driving signals necessary for a sensorless aberration correction implementation; and

- the possibility of having a common space between the assigned modes for correction to each adaptive element, which in contrast to explicitly separating the correction spaces, results in extending the amplitude range and improving the fidelity of the aberration correction.

\subsection{Approach}

Based on the hysteresis-free and linear (with respect to the square of the applied voltage) response of the DPP, we devised an optimization-based control system for calculating the driving signals of the DPP in open-loop operation, which is detailed elsewhere. ${ }^{27}$ Here, we extend this method for simultaneous control of the cascaded DPPs. The method is intended for replicating any arbitrary combination of Zernike modes using cascaded modulators and therefore can be employed for different $\mathrm{AO}$ applications where open-loop operation is a priority.

Given that the influence functions (the imprints of singular actuators with a unit control signal) of all electrodes are linearly independent, any arbitrary target surface shape can be represented as

$\mathbf{a}_{m}=\mathbf{B} \times \mathbf{c}$,

where $\mathbf{a}_{m}$ is a vector of length $M$ comprising the Zernike coefficients for the target membrane shape, $\mathbf{c}$ is a vector of electrode control signals ( $c_{l}=v_{l}^{2}$, where $v$ refers to voltage), and $\mathbf{B}$ is the empirical influence matrix. Here, $\mathbf{c}$ is of length $N$, where $N$ is the number of DPP electrodes. The influence matrix is of
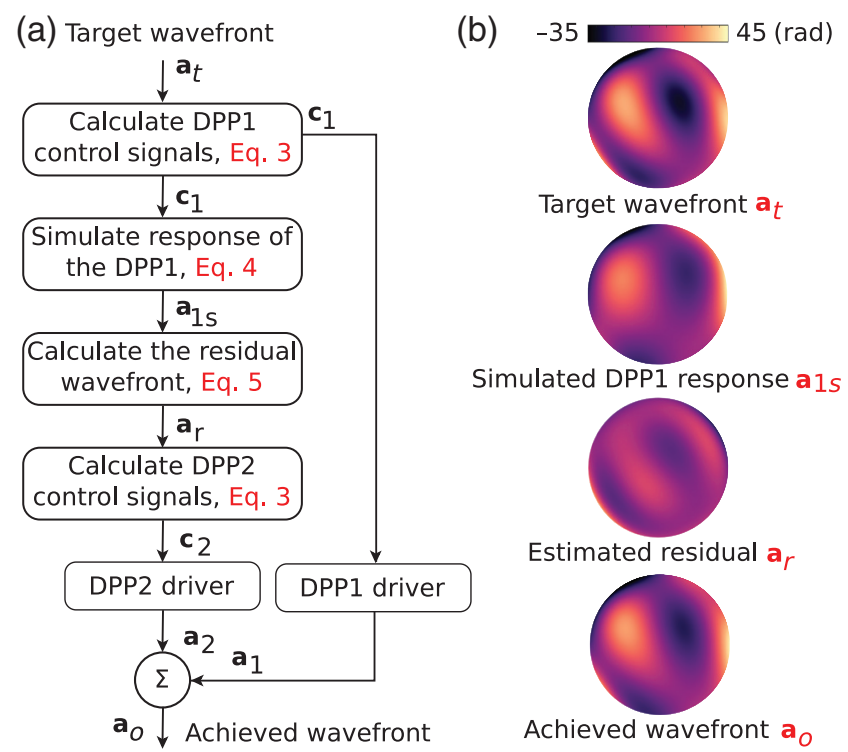

Fig. 3 Open-loop control method for simultaneous control of cascaded DPPs. (a) Control flowchart and (b) visualization of different steps of replicating a target wavefront.

dimensions $M \times N$, where each column of $\mathbf{B}$ corresponds to the empirically measured influence functions, represented again as a vector of Zernike coefficients. Hence, any element $b_{k l}$ of the matrix $\mathbf{B}$ is equal to the slope of the linear function relating the $k^{\prime}$ th Zernike coefficient to the control signal applied to the $l$ 'th electrode. ${ }^{27}$ The phase modulation associated with a membrane deformation $\mathbf{a}_{m}$ is given by

$\mathbf{a}=\mathbf{a}_{m}\left(n_{\text {fluid }}-n_{\text {air }}\right)$,

where $n_{\text {air }}$ and $n_{\text {fluid }}$ are the refractive indices of air and the optical liquid, respectively.

With Eq. (1), we can formulate the following optimal control problem for calculating the required vector of control signals $\mathbf{c}$ for replicating any arbitrary $\mathbf{a}$ :

$$
\begin{aligned}
\underset{\mathbf{c} \in \mathbb{R}^{P}}{\operatorname{minimize}} & f(\mathbf{c})=\frac{1}{2}\|\mathbf{B c}-\mathbf{a}\|_{2}^{2} \\
\text { subject to } & \mathbf{c} \geq 0, \\
& \mathbf{c} \leq \mathbf{c}_{\max } .
\end{aligned}
$$

This formulation treats the upper and lower boundaries of the control signals due to the electrostatic unipolar actuation and the maximum applicable voltage of the existing driving electronics as two inequality constraints. We have shown that this is a convex optimization problem and can be solved using an interior-point algorithm in real time. ${ }^{27}$ Being a convex problem, it is guaranteed that the obtained solution brings the optimal performance of the DPP for replicating any desired phase modulation.

The flowchart in Fig. 3(a) summarizes how the abovedescribed control method is extended for simultaneous driving of two DPPs. The final target wavefront $\mathbf{a}_{t}$ is used with Eq. (3) to calculate the control signals of DPP1, $\mathbf{c}_{1}$. Next, Eq. (1) is used for simulating the response of DPP1 with input $\mathbf{c}_{1}$ :

$\mathbf{a}_{1 s}=\mathbf{B}_{\mathrm{DPP} 1} \times \mathbf{c}_{1}$. 
Then, the simulated response is subtracted from the target wavefront to calculate the estimated residual after DPP1:

$\mathbf{a}_{\mathrm{res}}=\mathbf{a}_{t}-\mathbf{a}_{1 s}$.

In the next step, the residual wavefront is used as the target phase modulation for DPP2. Equation (3) is now used with the influence matrix of DPP2 for calculating its control signals $\mathbf{c}_{2}$ for replicating the residual wavefront $\mathbf{a}_{\text {res }}$. Finally, the control signals $\mathbf{c}_{1}$ and $\mathbf{c}_{2}$ for both DPPs are applied simultaneously to the DPPs. The overall replicated wavefront is the superposition of the responses of DPP1 and DPP2 $\left(\mathbf{a}_{o}=\mathbf{a}_{1}+\mathbf{a}_{2}\right)$. Figure 3(b) depicts one example of performing the proposed steps from the target to the achieved wavefront. In this work, we opted to start the algorithm by calculating the optimal control signals of DPP1, which has a lower actuator count. Alternatively, for a target aberration $\mathbf{a}_{t}$, Eq. (3) can be calculated for both DPP1 and DPP2 to compare their residual wavefront replication errors. Subsequently, the DPP with the lower residual error can be chosen as the initial modulator in the control algorithm.

Using a conventional PC with an Intel eighth-generation Core i7 processor, computation of control signals for both DPPs takes less than $10 \mathrm{~ms}$ when implemented in MATLAB. Since the optimization step is a convex problem, this method guarantees the best possible correction by the DPP1 that is used similar to a woofer for correcting the majority of the aberrations. DPP2 is used for (1) replicating any phase modulation that is beyond the capabilities of DPP1 (such as a tweeter), (2) improving the fidelity of the replicated wavefronts by compensating for the induced unwanted modes, and (3) extending the amplitude of the aberration correction even for lower orders. The proposed control algorithm is highly dependent on the estimation accuracy of the behavior of the phase modulators using their empirical model. Inaccuracies in this estimation can result in suboptimal performance and deviations in the target and achieved wavefronts.

\subsection{Proof-of-Concept of the Control Method}

As mentioned earlier, the accurate modeling of the DPP1 behavior is essential for a successful implementation of the proposed control method. To test this, we experimentally measured the response of DPP1 for replicating several target wavefronts and compared them with the estimations based on its response model. The cascaded DPPs are placed in the measurement arm of a custom-developed Mach-Zehnder interferometer. A computer equipped with a frame grabber captures the interferograms of $842 \times 842$ pixels, and the phase profiles are quantitatively extracted from the interferograms using the spatial-carrier fringepattern analysis technique. ${ }^{28,29}$ The measured profiles are fitted to Zernike polynomials using a total of 231 modes (up to the 20th radial order) for a precise fit. ${ }^{30}$

The bar plots in Fig. 4 present two examples of this test. The blue bars depict the Zernike decomposition of the target wavefront. In this work, we use the OSA/ANSI standard indexing scheme to represent the Zernike modes ${ }^{31}$ without normalization of the coefficients. Initially, the control signals of DPP1 are calculated using Eq. (3). The first overlying diagram on each plot shows the voltage distribution of the electrodes of DPP1.
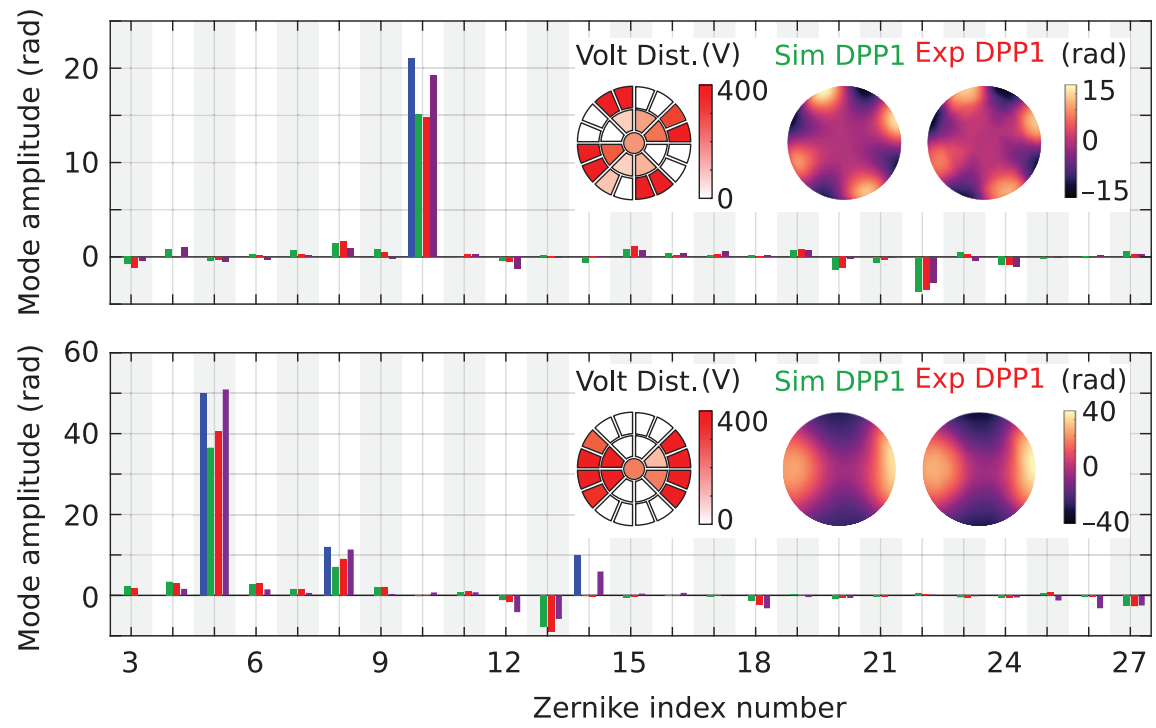

\begin{tabular}{|llll|}
\hline $\begin{array}{l}\text { Target } \\
\text { wavefront }\end{array}$ & $\begin{array}{l}\text { Simulated } \\
\text { DPP1 response }\end{array}$ & $\begin{array}{l}\text { Experimental } \\
\text { DPP1 response }\end{array}$ & $\begin{array}{l}\text { Experimental DPP1 } \\
\text { and DPP2 response }\end{array}$ \\
\hline
\end{tabular}

Fig. 4 Experimental evaluation of the introduced open-loop control for replicating two example wavefronts using DPP1 and DPP2. A target wavefront is chosen and the control signals of the DPP1 are calculated. The keystone patterns on each plot show the voltage distribution of the DPP1. These control signals are used to estimate the DPP1 response by its response model. The two wavefront profiles on each plot compare the estimated response from experimental measurements. Bar plots compare the Zernike decomposition of the target and reproduced wavefronts at each step of the control algorithm depicted in Fig. 3(a). Interferometric measurements are performed using a HeNe laser $(\lambda=632 \mathrm{~nm})$. 
The green bars show the simulated response of DPP1, while the red bars depict the measured response after the calculated control signals are applied. The Zernike coefficients of the obtained wavefronts from both the simulation and experiment for the target modes are smaller than the target values, meaning that the target amplitudes are beyond the correction capability of DPP1. Furthermore, the amplitudes of the unwanted modes are nonzero, degrading the fidelity of the wavefront replication.

Next, the residual phase between the target and the simulated response of DPP1 is calculated to obtain the control signals for DPP2. The violet bars show the overall measured wavefront with both DPPs on. The superposed wavefront matches the target one significantly better than those that could be obtained with individual DPPs.

The root-mean-square errors (RMSEs) between the estimated and experimentally measured wavefronts for the first (RMS = $4.91 \mathrm{rad}$ ) and second (RMS = $17.33 \mathrm{rad}$ ) sample wavefronts in Fig. 4 are 0.63 and $1.93 \mathrm{rad}$, respectively. This corresponds to an estimation error of $13 \%$ and $11 \%$. Although there is a slight difference between the simulated and experimental behavior of DPP1, the accuracy of the simulated response still makes the open-loop and simultaneous operation of DPPs possible without requiring a wavefront sensor. Furthermore, using this method, it is possible to control the cascaded DPPs such as a single adaptive element. The mentioned error will be magnified if the number of estimation steps increases (e.g., by cascading more than 2 DPPs). In the case of larger estimation errors, the proposed algorithm may not be the best alternative for driving the cascaded configuration. Choice of the most appropriate control strategy for the AO module is highly dependent on the exact conditions of the total system and requires a trade-off study.

\subsection{Zernike Mode Replication}

To investigate the performance of the cascaded configuration in replication of Zernike mode shapes, we used the same interferometer as in Sec. 3.2. As the first step, we tested the initial flatness and the best-flat of the cascaded DPPs. In the all off
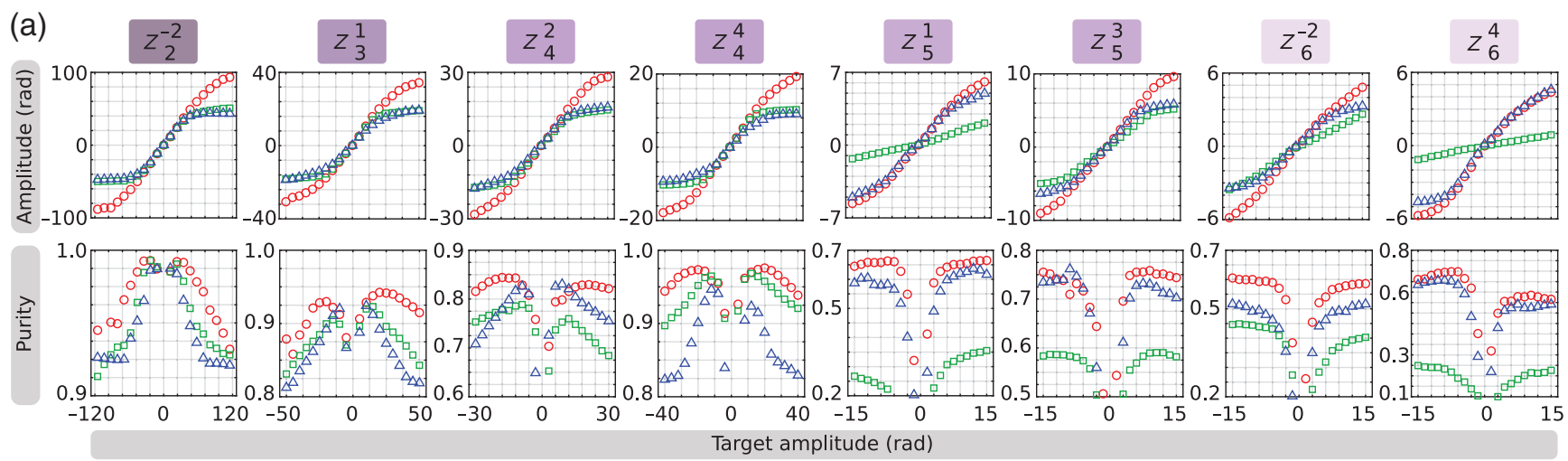

\section{\begin{tabular}{|lll|}
\hline DPP1 & $\triangle$ DPP2 & $\bigcirc$ DPP1 and DPP2 \\
\hline
\end{tabular}}

(b)

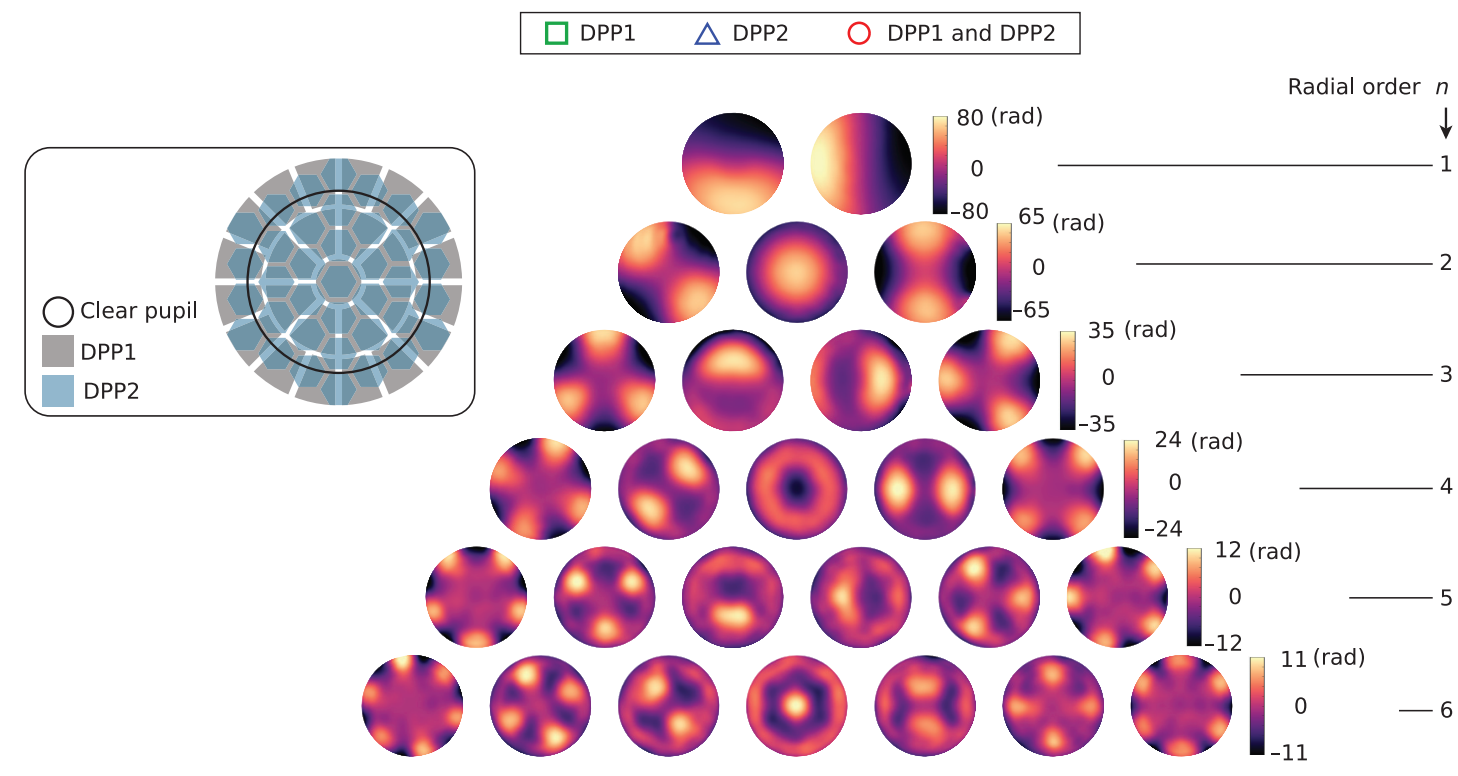

Fig. 5 Experimental results for replicating up to the sixth radial order of Zernike modes. (a) Comparing the maximum achievable mode amplitude and their corresponding purity [see Eq. (6)] using DPP1 and DPP2 individually (depicted by green squares and blue triangles, respectively), and simultaneously with the cascaded configuration and the proposed control method (red circles). (b) Overview of the wavefront profiles of the replicated Zernike modes using the cascaded DPPs. The top-left figure shows the overlay of DPP1 and DPP2 electrode patterns. Interferometric measurements are performed using a HeNe laser $(\lambda=632 \mathrm{~nm})$. 
condition, a wavefront error of 0.97 rad RMS was measured. After open-loop compensation of the initial membrane shapes of the DPPs, an active best-flat with an RMS value of $0.20 \mathrm{rad}$ was achieved. Next, the open-loop performance for replicating up to the sixth radial order of Zernike modes was tested. We considered the amplitude and the purity of the replicated modes as quality metrics. Purity is a unitless figure of merit between 0 and 1 that represents the fidelity of the replicated mode shapes, and is defined as

$$
P_{k}=\frac{a_{k}}{\sqrt{\sum_{i=0}^{M_{f}} a_{i}^{2}}}
$$

where $a_{k}$ corresponds to the coefficient of the target mode $k$, $i$ is the Zernike index number, and $M_{f}$ is the number of modes used for wavefront fitting (here, $M_{f}=231$ ). A purity of 1 indicates perfect mode reconstruction, where the contribution of the unwanted modes in the achieved wavefront is zero. Piston, tip, and tilt contributions are ignored for calculation of purity as they are not considered as optical aberrations.

Figure 5(a) plots the amplitude and purity of replicated modes as a function of the target mode amplitude until saturation for eight different modes in three configurations: DPP1-only (green squares), DPP2-only (blue triangles), and the cascaded DPPs together (red circles). The performance of each DPP based on its electrode pattern is different for different Zernike modes.

(a)

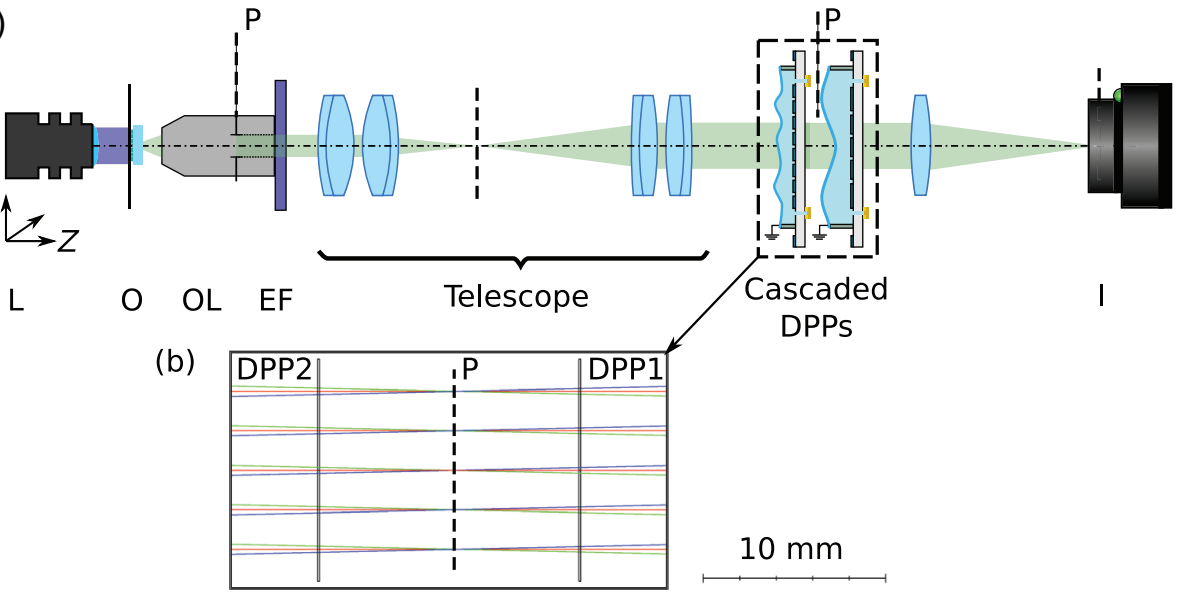

(c)

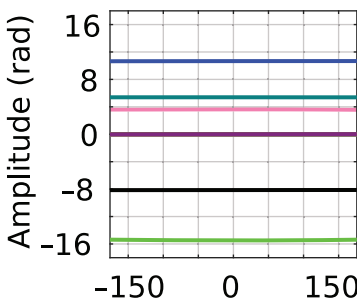

(d)

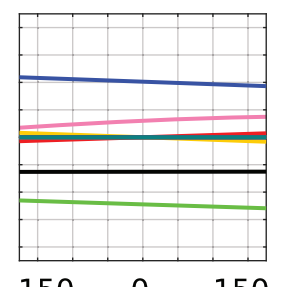

(e)

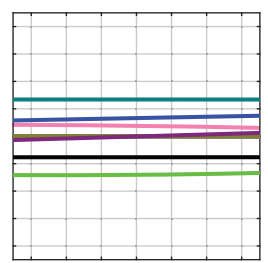

(f)

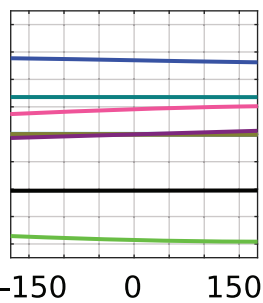

Field position $(\mu \mathrm{m})$

\begin{tabular}{|llllllll}
\hline \multirow{2}{*}{ Zernike modes } & $-z_{2}^{-2}$ & $-z_{2}^{0}$ & $-z_{2}^{2}$ & $-z_{3}^{-3}$ & $-z_{3}^{-1}$ & $-z_{3}^{1}$ & $-z_{3}^{3}$ \\
& $-z_{4}^{-4}$ & $-z_{4}^{-2}$ & $-z_{4}^{0}$ & $-z_{4}^{2}$ & $-z_{4}^{4}$ & $-z_{5}^{5}$
\end{tabular}

(g)

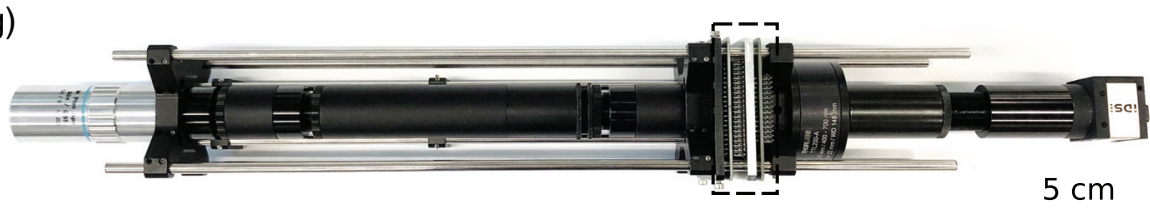

Fig. 6 (a) Schematic layout of the developed AO microscope. P, pupil plane; O, object; OL, objective lens; EF, emission filter; and I, image. (b) Detailed view of the ray-tracing simulation model at the location of cascaded DPPs each having a 7-mm offset from the conjugated pupil plane. Simulation results depicting the amplitudes of the Zernike modes with respect to field positions for (c) the ideal case of having both DPPs directly at the pupil plane, (d) only DPP1 actuated while being positioned $7 \mathrm{~mm}$ behind the pupil plane, (e) only DPP2 actuated while being positioned $7 \mathrm{~mm}$ in front of the pupil plane, and (f) DPP1 and DPP2 actuated simultaneously at their offsetposition. (g) Overview of the fully refractive AO microscope with the integrated cascaded DPPs. 
While DPP1 with 25 electrodes has a comparable performance with DPP2 with 37 electrodes for replicating the low-order Zernike modes (even for some modes better such as $Z_{4}^{4}$ ), DPP2 outperforms DPP1 for replicating high-order modes (e.g., see the characterization plots for $Z_{5}^{1}$ and $Z_{6}^{4}$ ). For the modes where both DPPs have a similar performance, the cascaded configuration increases the range of amplitudes by a factor of 2 . Furthermore, the purity of mode replication also improves for almost all the modes, achieved by compensating for the induced unwanted modes, which is an empirical validation of the openloop control method discussed in Sec. 3. The tree plot in Fig. 5(b) shows the experimentally measured Zernike mode replications using the cascaded system, up to the sixth radial order, together with an overlaid representation of the electrode distributions for both DPPs.

\section{Fluorescence AO Microscope with Cascaded DPPs}

To demonstrate the performance of the developed configuration within a functional imaging system, we employed the cascaded DPPs in a widefield fluorescence microscope designed for pupil-plane aberration correction. Figure 6(a) depicts the layout of this instrument, whose design and performance details can be found in Ref. 18. The microscope has a telescope with $2 \times$ magnification to relay the objective pupil plane (Mitutoyo, $50 \times, \mathrm{NA}=0.55$, working distance $=13 \mathrm{~mm}$ ) and adjust its diameter to the DPP clear aperture size. The total magnification of the microscope is $25 \times$. There is no dedicated wavefront sensor, and the wavefront errors are estimated using an image-based iterative algorithm, as discussed in Sec. 4.1. Figure 6(g) shows an image of the AO microscope with the cascaded DPPs located at the conjugate of the pupil plane.

For pupil-plane AO correction, the phase modulator(s) should be located exactly at plane(s) conjugate to the objective pupil such that all fields are modulated with the same wavefront profile. However, in our current implementation, there is a 14-mm distance between the deformable surfaces of the two DPPs due to the thickness of the fluidic ports and the printed circuit board used for electrical access to the DPPs. Thus, placed symmetrically with respect to the pupil conjugate plane, each modulator sits at a 7-mm axial offset from their ideal position. Using the ray tracing simulations, we analyzed the effect of this offset on the aberration correction across the field of view (FoV). The deformable surfaces of the DPPs were defined by a set of Zernike polynomials, and the objective was modeled as an ideal paraxial lens. Figure 6(b) shows the zoomed-in view of the two DPPs, while the conjugate pupil plane (the dashed line) is placed in the middle of the two.

To study the effect of the non-ideal DPP position on the field aberrations, we first chose a random phase profile $\mathbf{a}_{t}$ and numerically calculated the expected deformation of both DPPs to reproduce it. The calculated profiles were then applied to the DPPs in the ray tracing model, and the resulting aberrations are measured across the FoV and compared to the ideal case shown in Fig. 6(c), where both DPPs are exactly at the pupil plane. The $x$ axis of the plots depicts the field positions sampled in both the vertical and horizontal directions, and then averaged. As expected, the induced aberrations are constant over the entire FoV in the ideal case. When only DPP1, placed $7 \mathrm{~mm}$ behind the pupil plane, is deformed by $\mathbf{a}_{t}-\mathbf{a}_{\mathrm{DPP} 2}$, the resulting aberrations show a slight variation over the FoV, where spherical aberration (mode $Z_{4}^{0}$ ) remains constant [Fig. 6(d)]. When DPP1 is flat and only DPP2, placed $7 \mathrm{~mm}$ in front of the pupil plane, is deformed to replicate $\mathbf{a}_{t}-\mathbf{a}_{\mathrm{DPP} 1}$, the aberrations again vary over the FoV, but with an opposite slope [Fig. 6(e)]. When both DPP1 and DPP2 are deformed to replicate the total target aberration $\left(\mathbf{a}_{\text {DPP1 }}+\mathbf{a}_{\text {DPP2 }}=\mathbf{a}_{t}\right)$, the opposite slopes partially compensate each other, but the aberrations still vary over the FoV [Fig. 6(f)]. The deviation from the ideal case of having both DPPs at the pupil plane can be seen by comparing Figs. 6(c) and 6(f). The maximum variation, however, is limited to $\sim 0.003 \mathrm{rad} / \mu \mathrm{m}$. Since, with pupil-plane AO aberration, correction only works effectively within the isoplanatic patch, and thus the corrected FoV is usually limited, ${ }^{32}$ this variation can be speculated to be practically negligible.

\subsection{Sensorless Aberration Estimation}

Instead of a wavefront sensor, we used a variant of the well-known modal decomposition algorithm for aberration estimation. ${ }^{33}$ In this method, the aberrations are assumed to be a linear superposition of an orthonormal set of basis modes, such as Zernike polynomials, and an image quality metric is maximized for each mode sequentially. It is assumed that the image quality metric is a convex function with a Gaussian profile with respect to the aberration modes. Therefore, at least three images at three different amplitudes of each aberration mode are needed to obtain a fit to the merit function (MF) (e.g., by simple curve fitting). Subsequently, the required correction for maximizing the MF with respect to that aberration mode is estimated.

The phase modulator of the AO module is used to apply the known biases of aberration modes, and for each bias, the quality score of the captured image is calculated. Hence, open-loop control of the actuation signals of the adaptive element is crucial. The specific MF we used to quantify the quality of the captured images is based on the spatial frequency content and is calculated as ${ }^{34}$

$\mathrm{MF}=\int_{\theta=0}^{2 \pi} \int_{m=M_{1}}^{M_{2}} S_{J}(\mathbf{m}) m \mathrm{~d} m \mathrm{~d} \theta$,

Table 1 Parameters of the sensorless aberration estimation algorithm employed for the experiments presented in this paper. $M_{1}$ and $M_{2}$ depict the minimum and maximum of the normalized spatial frequencies, respectively, to be used for calculation of the MF using Eq. (7).

\begin{tabular}{|c|c|c|c|c|}
\hline Iteration No. & $\begin{array}{c}\text { Correcting } \\
\text { modes } \\
\text { (Zernike index) } \\
\end{array}$ & $M_{1}$ & $M_{2}$ & $\begin{array}{c}\text { Bias } \\
\text { amplitude }\end{array}$ \\
\hline 1 & $3,5,7,8$ & $1 \%$ & $5 \%$ & $25 \mathrm{rad}$ \\
\hline 2 & $3,5,7,8$ & $1 \%$ & $5 \%$ & $15 \mathrm{rad}$ \\
\hline 3 & $3,5,7,8,12$ & $1 \%$ & $10 \%$ & $10 \mathrm{rad}$ \\
\hline 4 & 3,5 to 14 & $1 \%$ & $35 \%$ & $5 \mathrm{rad}$ \\
\hline 5 & 3,5 to 14 & $1 \%$ & $35 \%$ & $5 \mathrm{rad}$ \\
\hline 6 & 3,5 to 14 & $1 \%$ & $35 \%$ & $1 \mathrm{rad}$ \\
\hline$\geq 7$ & 3,5 to 20 & $1 \%$ & $80 \%$ & $1 \mathrm{rad}$ \\
\hline
\end{tabular}


where $\theta$ and $m$ are the annular and radial coordinates in the spatial frequency domain, respectively, and $\mathbf{m}$ is the spatial frequency vector. $S_{J}(\mathbf{m})$ is the image spectral density equal to the absolute value of the Fourier transform of the image. $M_{1}<|\mathbf{m}|<M_{2}$ represents an annular spatial frequency range such that the MF has the maximum sensitivity with respect to aberrations.

We use several iterations of this algorithm with progressively decreasing bias amplitudes to improve the aberration estimation. Multiple iterations are particularly useful in case of aberrations with an RMS value larger than 1 rad. ${ }^{35}$ The result of each iteration is chosen as the initial condition of the next. Details of this implementation and its aberration estimation performance are discussed elsewhere. ${ }^{18}$ The parameters of the employed aberration estimation algorithm in this study are summarized in Table 1.

\subsection{Imaging Experiments}

As test objects, we used $1-\mu \mathrm{m}$ fluorescent beads (Polysciences, Fluoresbrite YG Microspheres $1 \mu \mathrm{m}$ ) with an emission wavelength of $485 \mathrm{~nm}$. A laser diode with a center wavelength of $405 \mathrm{~nm}$ (Roithner LaserTechnik, RLDE405-12-6) was used to excite fluorescent beads with flood illumination from the bottom. To mimic typical optical aberrations experienced in deep tissue imaging, we placed a custom phase plate directly on the microscope slide containing the micro-beads as an aberrating layer. For this, we used different locations of a poly(methyl methacrylate) foil (average thickness $375 \mu \mathrm{m}$ ) with random bending.

Figure 7 presents images acquired at four different sample positions without AO [Fig. 7(a)], with AO using only DPP1 [Fig. 7(b)], and with AO using both DPPs [Fig. 7(c)]. For both correction cases, the values of the quality MF after each (a) Without AO
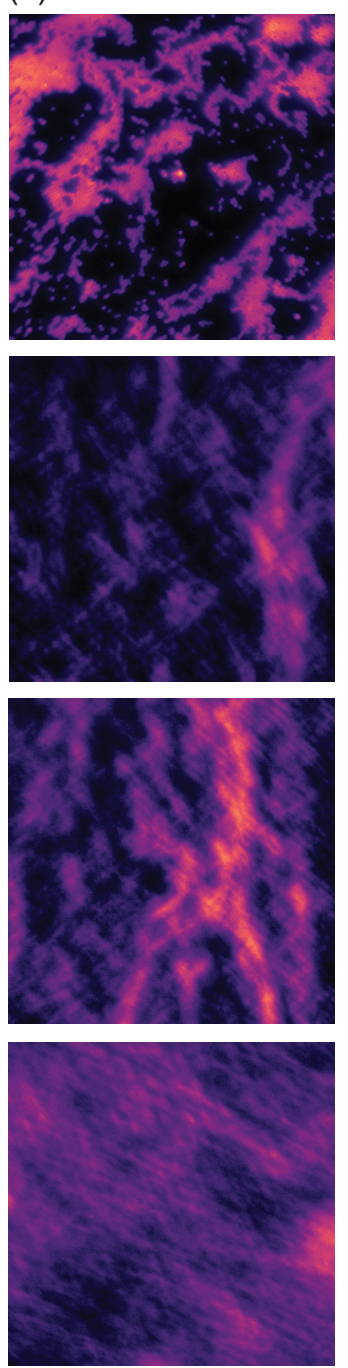

(b) With AO (DPP1)
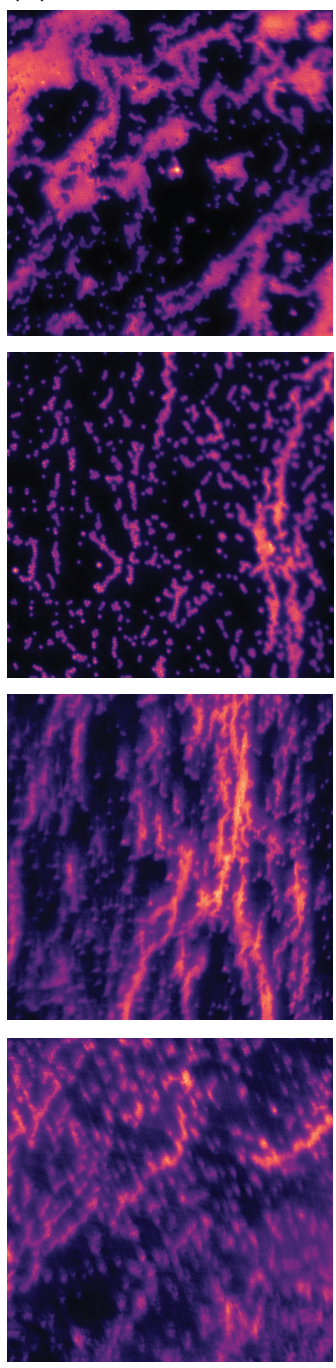

(C) With AO (DPP1 and DPP2)
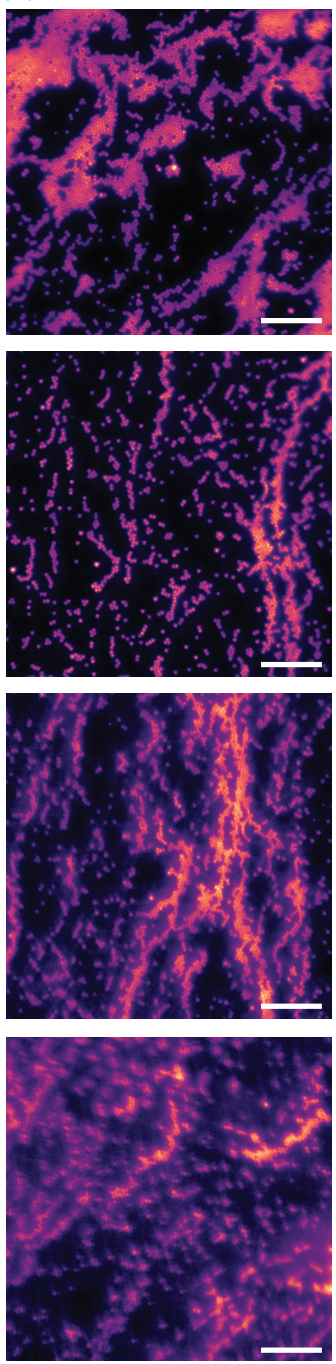

(d)
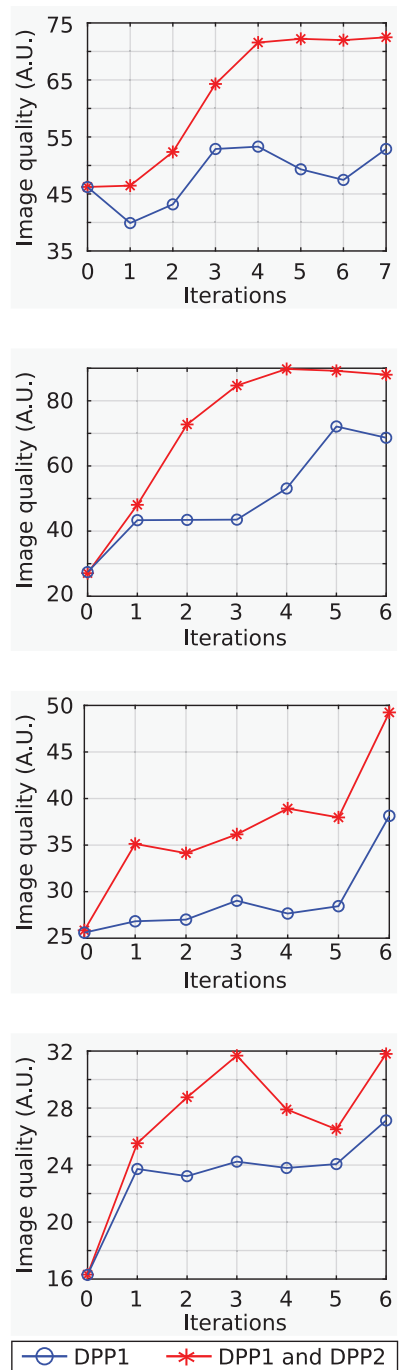

(e) Estimated aberration with both DPPs

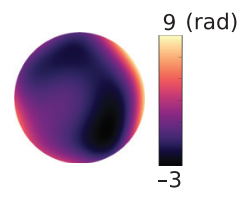

RMSE $=2.1 \mathrm{rad}$

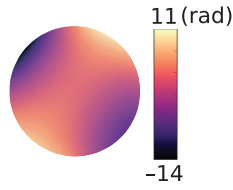

$\mathrm{RMSE}=4.1 \mathrm{rad}$

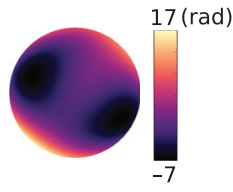

RMSE $=4.5 \mathrm{rad}$

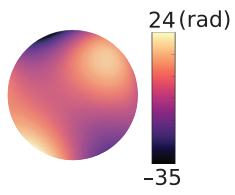

$\mathrm{RMSE}=9.7 \mathrm{rad}$

Fig. 7 Imaging fluorescent micro-beads $(\varnothing=1 \mu \mathrm{m})$ behind an aberrating phase plate that mimics specimen-induced aberrations while deep tissue imaging. (a) Aberrated frames, corrected frames using (b) a single DPP and (c) the cascaded configuration, (d) quality score of the obtained frames after each iteration of the aberration estimation algorithm, and (e) the estimated wavefront error by executing the sensorless aberration estimation algorithm using stacked DPPs. Length of the scale bars is $20 \mu \mathrm{m}$. 
iteration, calculated using identical spectral frequency ranges, are plotted in Fig. 7(d). The final estimated wavefront error and its RMS value for each aberration condition are presented in Fig. 7(e). The results demonstrate that, for the cascaded configuration, the corrected images have a better quality and the sensorless algorithm converges faster. The reason for these improvements is two-fold: (1) possibility of applying larger biases, which helps in faster identification of the required large amplitude corrections and (2) better purity of the applied modes.

One of the known causes for the suboptimal performance of the aberration estimation algorithms is the crosstalk between the influence of different aberration modes on the value of the image quality metric. This crosstalk, which is the main reason for requiring several iterations of this algorithm instead of only one iteration, ${ }^{18,35}$ is magnified in case of applying biases of distorted aberration modes. Therefore, the improved purity of the modes using the cascaded configuration helps in reducing the mentioned crosstalk and thus helps in faster convergence of the algorithm.

The initial iterations of the algorithm are performed by applying large amplitude biases. However, large biases are not suitable for high accuracy estimation of the low magnitude aberrations, which require small amplitude biases close to the final value. This explains why the image quality does not improve considerably in the initial iterations when the aberrations are small.

\section{Conclusion}

We have shown the implementation and performance of a new type of AO system employing two cascaded optofluidic phase modulators with contrasting electrode counts and arrangements. An optimization-based control scheme is employed for open-loop and simultaneous operation of both phase modulators for a large range of wavefront aberrations. Compared to the typical single modulator configuration, the proposed system significantly improves the available stroke and wavefront correction fidelity both for low- and high-order modes. The impact of this improvement has been empirically demonstrated in a wide-field fluorescence microscope using synthetic samples. Exceptionally compact and refractive, and capable of attaining significant correction amplitudes up to the sixth radial order Zernike modes, the presented AO system can be adapted to many applications including, but not limited to, life-science microscopy, ophthalmology, and beam shaping.

\section{Disclosures}

The authors declare no conflicts of interest.

\section{References}

1. R. Tyson, Principles of Adaptive Optics, CRC Press, Boca Raton, Florida (2010).

2. J. A. Kubby, Adaptive Optics for Biological Imaging, CRC Press, Boca Raton, Florida (2013).

3. P.-Y. Madec, "Overview of deformable mirror technologies for adaptive optics and astronomy," Proc. SPIE 8447, 844705 (2012).

4. S. Hu et al., "Double-deformable-mirror adaptive optics system for phase compensation," Appl. Opt. 45(12), 2638-2642 (2006).

5. R. J. Zawadzki et al., "Adaptive optics-optical coherence tomography: optimizing visualization of microscopic retinal structures in three dimensions," J. Opt. Soc. Am. A 24(5), 1373-1383 (2007).
6. W. Zou, X. Qi, and S. A. Burns, "Woofer-tweeter adaptive optics scanning laser ophthalmoscopic imaging based on Lagrangemultiplier damped least-squares algorithm," Biomed. Opt. Express 2(7), 1986-2004 (2011).

7. Q. Li et al., "Woofer-tweeter adaptive optical structured illumination microscopy," Photonics Res. 5(4), 329-334 (2017).

8. J. D. Barchers, "Closed-loop stable control of two deformable mirrors for compensation of amplitude and phase fluctuations," J. Opt. Soc. Am. A 19(5), 926-945 (2002).

9. M. R. Allen, J. J. Kim, and B. N. Agrawal, "Correction of an active space telescope mirror using a deformable mirror in a woofertweeter configuration," J. Astron. Telesc. Instrum. Syst. 2(2), 029001 (2016).

10. D. J. Wahl et al., "Wavefront sensorless adaptive optics fluorescence biomicroscope for in vivo retinal imaging in mice," Biomed. Opt. Express 7(1), 1-12 (2016).

11. R. D. Simmonds et al., "Three dimensional laser microfabrication in diamond using a dual adaptive optics system," Opt. Express 19(24), 24122-24128 (2011).

12. X. Lei et al., "Double-deformable-mirror adaptive optics system for laser beam cleanup using blind optimization," Opt. Express 20(20), 22143-22157 (2012).

13. A. J. Wright et al., "Dynamic closed-loop system for focus tracking using a spatial light modulator and a deformable membrane mirror," Opt. Express 14(1), 222-228 (2006).

14. K. Banerjee et al., "Optofluidic adaptive optics," Appl. Opt. 57(22), 6338-6344 (2018).

15. A. Tanabe et al., "Transmissive liquid-crystal device for correcting primary coma aberration and astigmatism in biospecimen in twophoton excitation laser scanning microscopy," J. Biomed. Opt. 21(12), 121503 (2016).

16. S. Bonora et al., "Wavefront correction and high-resolution in vivo OCT imaging with an objective integrated multi-actuator adaptive lens," Opt. Express 23(17), 21931-21941 (2015).

17. K. Philipp et al., "Diffraction-limited axial scanning in thick biological tissue with an aberration-correcting adaptive lens," Sci. Rep. 9(1), 9532 (2019).

18. P. Rajaeipour et al., "Fully refractive adaptive optics fluorescence microscope using an optofluidic wavefront modulator," Opt. Express 28(7), 9944-9956 (2020).

19. H. R. Verstraete et al., "Wavefront sensorless adaptive optics OCT with the DONE algorithm for in vivo human retinal imaging," Biomed. Opt. Express 8(4), 2261-2275 (2017).

20. J. M. Bueno et al., "Wavefront correction in two-photon microscopy with a multi-actuator adaptive lens," Opt. Express 26(11), 14278-14287 (2018).

21. K. Banerjee et al., "Refractive opto-fluidic wavefront modulator with electrostatic push-pull actuation," Proc. SPIE 10886, 108860D (2019).

22. C. Correia et al., "Minimum-variance control for woofer-tweeter systems in adaptive optics," J. Opt. Soc. Am. A 27(11), A133A144 (2010)

23. R. Conan et al., "Distributed modal command for a twodeformable-mirror adaptive optics system," Appl. Opt. 46(20), 4329-4340 (2007).

24. J.-F. Lavigne and J.-P. Véran, "Woofer-tweeter control in an adaptive optics system using a Fourier reconstructor," J. Opt. Soc. Am. A 25(9), 2271-2279 (2008).

25. W. Zou, X. Qi, and S. A. Burns, "Wavefront-aberration sorting and correction for a dual-deformable-mirror adaptive-optics system," Opt. Lett. 33(22), 2602-2604 (2008).

26. C. Li et al., "A correction algorithm to simultaneously control dual deformable mirrors in a woofer-tweeter adaptive optics system," Opt. Express 18(16), 16671-16684 (2010).

27. P. Rajaeipour et al., "Optimization-based real-time open-loop control of an optofluidic refractive phase modulator," Appl. Opt. 58(4), 1064-1072 (2019). 
28. D. Malacara, Optical Shop Testing, Vol. 59, John Wiley \& Sons (2007).

29. P. Rajaeipour et al., "Optimization-based open-loop control of phase modulators for adaptive optics," Proc. SPIE 10886, 108861A (2019).

30. D. J. Fischer et al., "Vector formulation for interferogram surface fitting," Appl. Opt. 32(25), 4738-4743 (1993).

31. L. N. Thibos et al., "Standards for reporting the optical aberrations of eyes," J. Refractive Surg. 18(5), S652-S660 (2002).

32. J. Mertz, H. Paudel, and T. G. Bifano, "Field of view advantage of conjugate adaptive optics in microscopy applications," Appl. Opt. 54(11), 3498-3506 (2015).

33. M. J. Booth et al., "Adaptive aberration correction in a confocal microscope," Proc. Natl. Acad. Sci. U.S.A. 99(9), 5788-5792 (2002).

34. D. Débarre, M. J. Booth, and T. Wilson, "Image based adaptive optics through optimisation of low spatial frequencies," Opt. Express 15(13), 8176-8190 (2007).

35. A. Facomprez, E. Beaurepaire, and D. Débarre, "Accuracy of correction in modal sensorless adaptive optics," Opt. Express 20(3), 2598-2612 (2012).

Pouya Rajaeipour received his BSc degree in mechanical engineering from Isfahan University of Technology and his MSc degree in microsystems engineering from the University of Freiburg, in 2015 and 2017, respectively. $\mathrm{He}$ is currently a $\mathrm{PhD}$ candidate at the Gisela and Erwin Sick Laboratory of Micro-Optics, the University of Freiburg in Germany. The focus of his research is on refractive $A O$ systems.

Kaustubh Banerjee earned his BSc degree in mechanical engineering from UCLA in 2012 followed by MSc and PhD degrees in microsystems engineering from the University of Freiburg in 2014 and 2020, respectively. He is a researcher at the laboratory of Micro-Optics, the University of Freiburg, Germany. His work focuses on developing optofluidic devices with particular applications for $\mathrm{AO}$.

Alex Dorn received his BSc and MSc degrees both in microsystems engineering from Albert Ludwig University of Freiburg with a focus on optofluidics and microscopy, in 2017 and 2019, respectively. Currently, he is a PhD student with the main research emphasis realizing novel optofluidic AO solutions for microscopy.

Hans Zappe is Gisela and Erwin Sick Professor of Micro-Optics at the University of Freiburg in Germany. He earned BSc and MSc degrees from MIT in 1983 and a PhD from the University of California, Berkeley, in 1989, all in electrical engineering. He worked at IBM (USA), the Fraunhofer Institute for Applied Solid State Physics (Germany), and the Centre Suisse d'Electronique et de Microtechnique (Switzerland), before joining the Department of Microsystems Engineering at the University of Freiburg in 2000. He was dean of engineering from 2008 to 2010 , dean of studies from 2002 to 2008 , and currently serves as the department chair. $\mathrm{He}$ is a fellow of SPIE, OSA, and IOP.

Çağlar Ataman received his $\mathrm{PhD}$ in electrical engineering from Koç University (Istanbul, Turkey) in 2008, and worked as a post-doctoral researcher at École Polytechnique Fédérale de Lausanne (Switzerland) between 2008 and 2012. Since 2012, he is a senior scientist and a group leader in the Micro-Optics Laboratory at the University of Freiburg (Germany). His current research focuses on optical microsystems for biomedical imaging and life-science microscopy. 